\title{
Influence of domain size on optical properties of ordered Galn $\mathbf{P}_{\mathbf{2}}$
}

\author{
P. Ernst, ${ }^{\text {a) }}$ C. Geng, G. Hahn, F. Scholz, and H. Schweizer \\ 4. Physikalisches Institut, Universität Stuttgart, Pfaffenwaldring 57, 70550 Stuttgart, Germany \\ F. Phillipp \\ Max-Planck-Institut für Metallphysik, Heisenbergstrasse 1, 70569 Stuttgart, Germany
}

\author{
A. Mascarenhas \\ National Renewable Energy Laboratory, 1617 Cole Boulevard, Golden, Colorado 80401
}

(Received 13 September 1995; accepted for publication 16 November 1995)

\begin{abstract}
Using dark-field transmission electron microscopy images of ordered GaInP samples, we show how the ordering domain size depends on the growth temperature. Samples with different average domain sizes are compared with regard to their photoluminescence (PL) and excitation spectra. We find a close correlation between the size of the ordered domains and the relative intensity of the PL peak from band-band recombination compared with the rapidly shifting, below-band-gap luminescence emission. (C) 1996 American Institute of Physics. [S0021-8979(96)06304-5]
\end{abstract}

\section{INTRODUCTION}

$\mathrm{GaInP}_{2}$, grown lattice matched on GaAs substrates by metal-organic vapor-phase epitaxy (MOVPE), has achieved considerable interest in both basic research and commercial application. Due to its high direct band gap, the material is used in red-light-emitting laser diodes and light-emitting diodes (LEDs) $)^{1-3}$ as well as for high-efficiency solar cells. ${ }^{4}$ The research activities are focused on the phenomenon of spontaneous long-range ordering of the group-III constituents $\mathrm{Ga}$ and $\mathrm{In}$ on a monolayer superlattice oriented in a [111] direction during the growth process. It has been shown that this type of $\mathrm{CuPt}_{B}$ ordering occurs in many III-V compounds if appropriate growth conditions are chosen (for a recent overview see Ref. 5). Nevertheless, most of the experimental work carried out so far concentrated on the $\mathrm{GaInP}_{2}$ compound. This is because of the fact that relatively strong ordering occurs in this alloy at growth conditions that are very close to those leading to the highest-quality material in MOVPE (i.e., growth temperatures of about $750{ }^{\circ} \mathrm{C}$ ). It appears that almost every GaInP/AlGaInP alloy used for optoelectronic devices shows some degree of $\mathrm{CuPt}_{B}$ ordering. Therefore, knowledge about material parameters that depend on the degree of order is also useful for the design of devices.

It has been shown theoretically and experimentally, that ordering causes a reduction of the direct band gap and a splitting of the valence band at the $\Gamma$ point. Both effects are a direct result of the reduced symmetries of the (ordered) $\mathrm{CuPt}_{B}$ structure compared with the (disordered) zinc-blende structure. The degree of enrichment of Ga and In in the alternating planes determines the interaction of bands of equal symmetry (level repulsion of zone-folded states). This explains the continuous increase of both the reduction of the band gap (mainly due to a depression of the $\Gamma_{6}^{C}$ conduction band) and the splitting of the valence band from the degenerate $\Gamma_{8}^{V}$ (zinc blende) to $\Gamma_{4,5}^{V}$ and $\Gamma_{6}^{V}(2)\left(\mathrm{CuPt}_{B}\right){ }^{6-8}$

\footnotetext{
a) Present address: National Renewable Energy Laboratory, 1617 Cole Boulevard, Golden, CO 80401.
}

These changes in the intrinsic band structure of the ordered material are well understood theoretically and are confirmed by optical spectroscopy. ${ }^{9-14}$ Nevertheless, some more properties concerning the microstructure of ordered $\mathrm{GaInP}_{2}$ are still unclear. In particular, low-temperature photoluminescence (PL) spectra are dominated by a peak that comes from below-band-gap transitions $(10 \ldots 50 \mathrm{meV}$ below the fundamental band gap) and moves to higher emission energy with increasing excitation intensity ("moving" emission). Previous work ${ }^{15-20}$ has established the following facts about this PL feature:

(1) It is not due to usual donor or acceptor states (impurities) with well-defined binding energies, because

(a) its dependence on excitation intensity is much stronger than expected for a donor- or acceptor-related optical transition, ${ }^{21,22}$ and

(b) it vanishes with decreasing order parameter, ${ }^{20}$ although an impurity would also appear in the disordered material.

(2) There is no absorption of light in the energy range of the moving emission. Instead, PLE spectra monitored with detection energies positioned at the moving emission show the same band edge as those monitored on the free exciton. ${ }^{20}$

(3) The lifetime of the moving emission is extremely long and depends strongly on the detection energy. ${ }^{16,17,20}$

(4) With increasing temperature, the moving emission first shifts to higher energy, then loses intensity compared with the direct band-gap PL, and finally disappears completely. ${ }^{15,19}$ At room temperature, only direct band-band recombination is observed, as expected from any semiconductor with a direct band gap.

On the other hand, transmission electron microscopy (TEM) studies have shown that the microstructure of ordered $\mathrm{GaInP}_{2}$ often consists of domains of ordered material, embedded in a more disordered matrix. ${ }^{23,24}$ The size of the ordered domains seen in dark-field images strongly depends on growth conditions and may range from $10 \mathrm{~nm}$ up to $1 \mu \mathrm{m}$ (lateral width). ${ }^{25,26}$ 


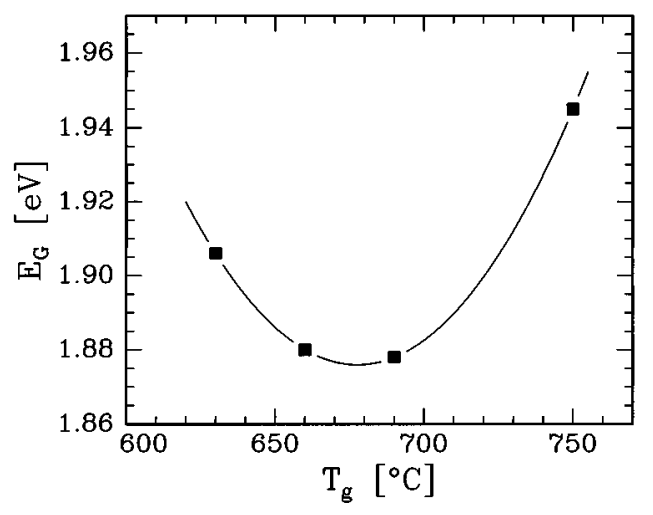

FIG. 1. Solid squares: Band-gap energy of each of the samples investigated, determined by low-temperature PLE. We have used the maximum of the first excitonic resonance as a measure for the band gap $E_{G}$, without correcting for the excitonic binding energy. Solid line: Parabola, fitted to the data as a guide to the eye.

The aim of this investigation is to show

(1) how the domain size depends on the growth temperature, which is the only growth parameter varied here, and

(2) that there is a close correlation between the average domain size in an ordered sample and its optical properties as measured by low-temperature PL and excitation spectroscopy (PLE).

\section{EXPERIMENT}

\section{A. Samples}

We have grown a series of four samples of bulk $\mathrm{GaInP}_{2}$ by means of low-pressure MOVPE, described in more detail in Ref. 20. The degree of ordering was controlled by varying the growth temperature between 630 and $750{ }^{\circ} \mathrm{C}$. Each sample was grown on a (100) GaAs substrate misoriented $6^{\circ}$ off toward $[111]_{B}$. The growth rate was $2.0 \mu \mathrm{m} / \mathrm{h}$ at a V/III ratio of 240 . The layer thickness of each sample is $2 \mu \mathrm{m}$.

We have determined the band gap of the samples by low-temperature PLE (see Sec. III). Figure 1 shows a plot of the band-gap energy of the samples versus growth temperature $T_{g}$. The solid line is a parabola fitted to the data as a guide to the eye. It can be seen that the growth temperatures of the samples cover the range of maximum ordering (the temperature of the highest degree of order $T_{g}^{\mathrm{max}}$ is probably about $680^{\circ} \mathrm{C}$. It is shown in Sec. III that there is a fundamental difference between samples with $T_{g}<T_{g}^{\max }$ and samples with $T_{g}>T_{g}^{\max }$.

\section{B. Transmission electron microscopy}

The TEM investigations have been performed using [011] cross-section slides. The slides have been prepared by gluing two GaInP surfaces together (in a stack of $\mathrm{Si}$ wafer pieces for better stability). Disks with a thickness of $500 \mu \mathrm{m}$ were cut perpendicularly to the layers and mechanically polished to a thickness of $100 \mu \mathrm{m}$. Then, hemispherical dimples were created on the glue line by means of a rotating copper wheel (dimpler). A second chemical polishing was carried out in a solution of $\mathrm{H}_{2} \mathrm{SO}_{4}: \mathrm{H}_{2} \mathrm{O}_{2}: \mathrm{H}_{2} \mathrm{O}(3: 1: 1)$ for $60 \mathrm{~s}$ at a temperature of $43{ }^{\circ} \mathrm{C}$. Finally, the slides were thinned in an $\mathrm{Ar}^{+}$beam at the temperature of liquid nitrogen.

The images have been taken with the electron beam oriented along the crystal's [011] direction, with a spot size on the sample of about $1 \mu \mathrm{m}$ for diffraction patterns and $5 \mu \mathrm{m}$ for dark-field (DF) images. The dark-field images have been taken using the $\frac{1}{2}(3 \overline{1} 1)$ reflex, because the $\frac{1}{2}(1 \overline{1} 1)$ reflex yielded images with a poor contrast caused by some background intensity of the direct beam.

\section{Optical spectroscopy}

Optical measurements were performed using an argonion laser operated in multiline mode (with main laser lines at 488 and $514 \mathrm{~nm}$ ) for PL and a tunable dye laser filled with DCM (available wavelength range: $610 \ldots 700 \mathrm{~nm}$ ) for PLE. Luminescence was dispersed by a $0.85 \mathrm{~m}$ double monochromator and detected by a cooled InGaAs photomultiplier.

\section{RESULTS}

Figure 2 shows the diffraction patterns [transmission electron diffraction (TED), left-hand side] and the corresponding DF images (DF TEM), right-hand side) of the samples under investigation. Each of the diffraction patterns shows intense superlattice reflections of only one order plane orientation. This confirms the well-known fact ${ }^{24}$ that growth on a substrate misoriented toward a $[111]_{B}$ direction results in single-variant samples. The TED picture of the sample grown at $630{ }^{\circ} \mathrm{C}$ shows superlattice spots elongated along the [100] direction, with streaks slightly tilted off the exact [100] orientation. This has been attributed

(1) to a platelike shape of the ordering domains oriented with their longer axis nearly perpendicular to the growth direction, ${ }^{24,27}$ or

(2) to closely spaced "order twin boundaries" separating volumes of different order plane orientation. ${ }^{26}$

However, the latter explanation does not hold for the samples investigated here, because the second-order plane variant is not present (see TED images). As can be seen in the DFTEM images, the reason for the elongated superlattice spots is the smallness of the ordered domains. Due to a sufficient domain size, the superlattice spots from samples (b), (c), and (d) are perfectly circular.

The main feature of the series of DF-TEM pictures on the right-hand side of Fig. 2 is the important change in domain size with varying growth temperature. The order domains are extended in a direction almost parallel to a (111) plane in the case of samples (c) and (d), and are nearly parallel to the growth direction in samples (a) and (b). The lateral domain size varies systematically from about $10 \mathrm{~nm}$ in sample (a) to $\sim 500 \mathrm{~nm}$ in sample (d) (Fig. 3).

Now we turn to the optical data obtained from these samples. Figure 4 shows both PL (dashed) and PLE spectra (solid), taken at $T=2 \mathrm{~K}$. All spectra are plotted on the same energy scale to enable a direct comparison of peak energies and linewidths. The PL spectra are taken with an excitation intensity of $5 \mathrm{~W} / \mathrm{cm}^{2}$. 
(a)

$T_{g}=630{ }^{\circ} \mathrm{C}$

(b)

$T_{g}=660{ }^{\circ} \mathrm{C}$

(c)

$T_{g}=690{ }^{\circ} \mathrm{C}$
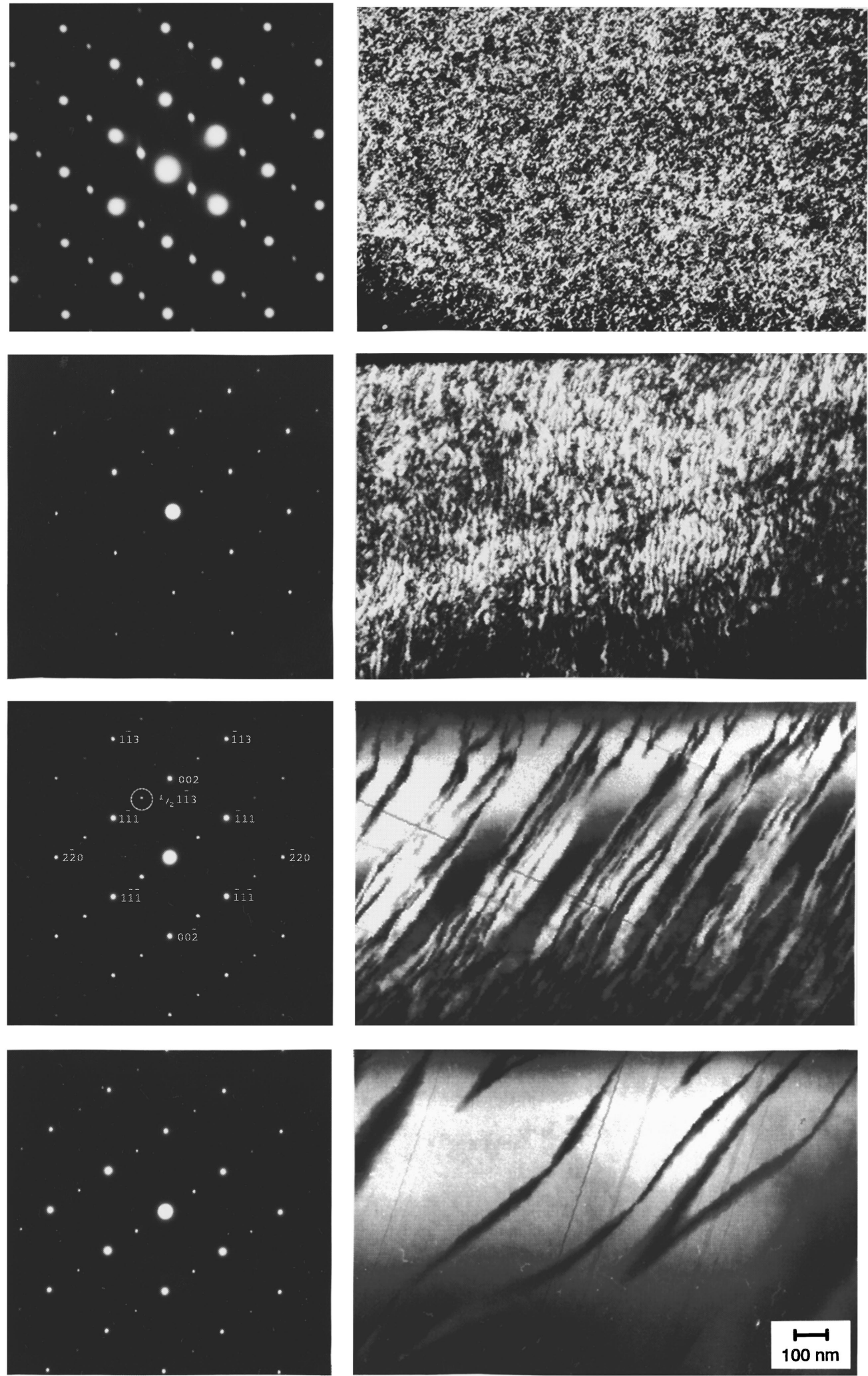

(d)

$T_{g}=750{ }^{\circ} \mathrm{C}$

FIG. 2. TED patterns (left-hand side) and DF-TEM images (right-hand side) of the samples grown at $630,660,690$, and $750{ }^{\circ} \mathrm{C}$. The superlattice spot that has been used to create the DF images is marked by a white circle in (c), left-hand side. The superspot labels in this image, however, differ from those used in the text. In the figure we use the denominations common in electron microscopy that assume a [110] cross-section slide of a sample with a (001) growth direction. 


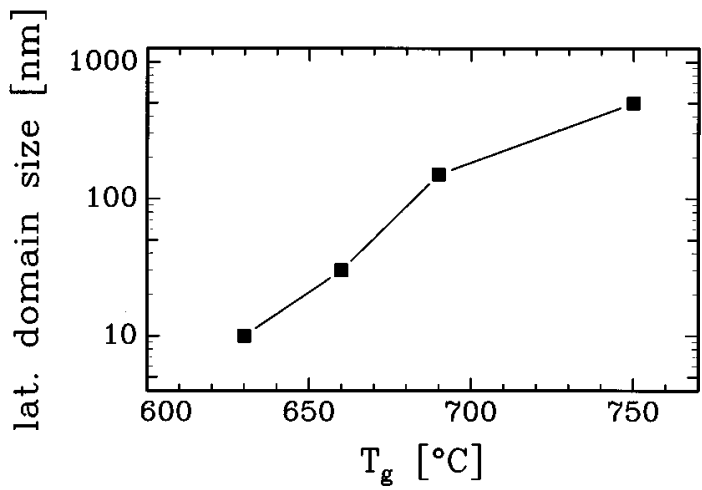

FIG. 3. Typical lateral domain size of the samples as a function of the growth temperature $T_{g}$.

The samples grown at 750 and $690{ }^{\circ} \mathrm{C}$ [samples (c) and (d)] show two PL peaks. The low-energy (LE) PL peak of both samples moves to higher emission energies with increasing excitation intensity [by (c) $5.0 \mathrm{meV} / \mathrm{dec}$ ade and by (d) $1.3 \mathrm{meV} /$ decade], whereas the high-energy (HE) peak is stationary. The linewidths of the HE peaks are 6.8 and 5.0 $\mathrm{meV}$ for samples (c) and (d), respectively, and the linewidths of the LE peaks are (c) $10.7 \mathrm{meV}$ and (d) $8.5 \mathrm{meV}$. In sample (d) the HE peak is dominant, whereas this peak is quite weak in sample (c). The PLE spectra A and B are monitored at the positions indicated by arrows, respectively. Excitonic absorption resonances correlated with both valence-band edges are clearly resolved in samples (d) and (c); their energetic positions are indicated by dashed-dotted lines. Their separation corresponds to the order-induced valence-band splitting. ${ }^{14}$ Comparison of PL and PLE spectra points to the fact that the HE peak represents the intrinsic (excitonic) band-band recombination [the Stokes shifts for samples (c) and (d) are 8.8 and $4.5 \mathrm{meV}$, respectively]. The origin of the (moving) PL peaks, on the other hand, is still unclear.

We now turn to samples (a) and (b), grown at lower temperatures. Here the (excitonic) HE emission vanishes and the single 15-17 meV broad PL line shows all the features of the above-mentioned LE peak: It moves with increasing excitation intensity, it shows long, detection-energy-dependent lifetimes, and it shows the inverted- $S$ behavior. ${ }^{20}$ PLE spectra, detected at the peak maximum and at the LE flank, show smoothly rising flanks without sharp excitonic maxima. Nevertheless, the separation of the two valence-band edges can be estimated (see the dashed-dotted lines).

So far, we conclude that the PL intensity of the intrinsic HE luminescence peak compared with the LE peak decreases with decreasing domain size (see Figs. 2 and 3), and even vanishes completely at a critical domain size (lying between 30 and $150 \mathrm{~nm}$ ).

However, it could be argued that the extinction of the intrinsic recombination in some samples could be due to a poor overall sample quality, e.g., high impurity concentrations, rather than the domain size. Therefore, we have made the following experiment on sample (c): It can be seen from Fig. 2 [(c), right-hand side] that the size of the ordered domains varies throughout the layer. It is largest near the sample surface (top), and becomes smaller closer to the sub-

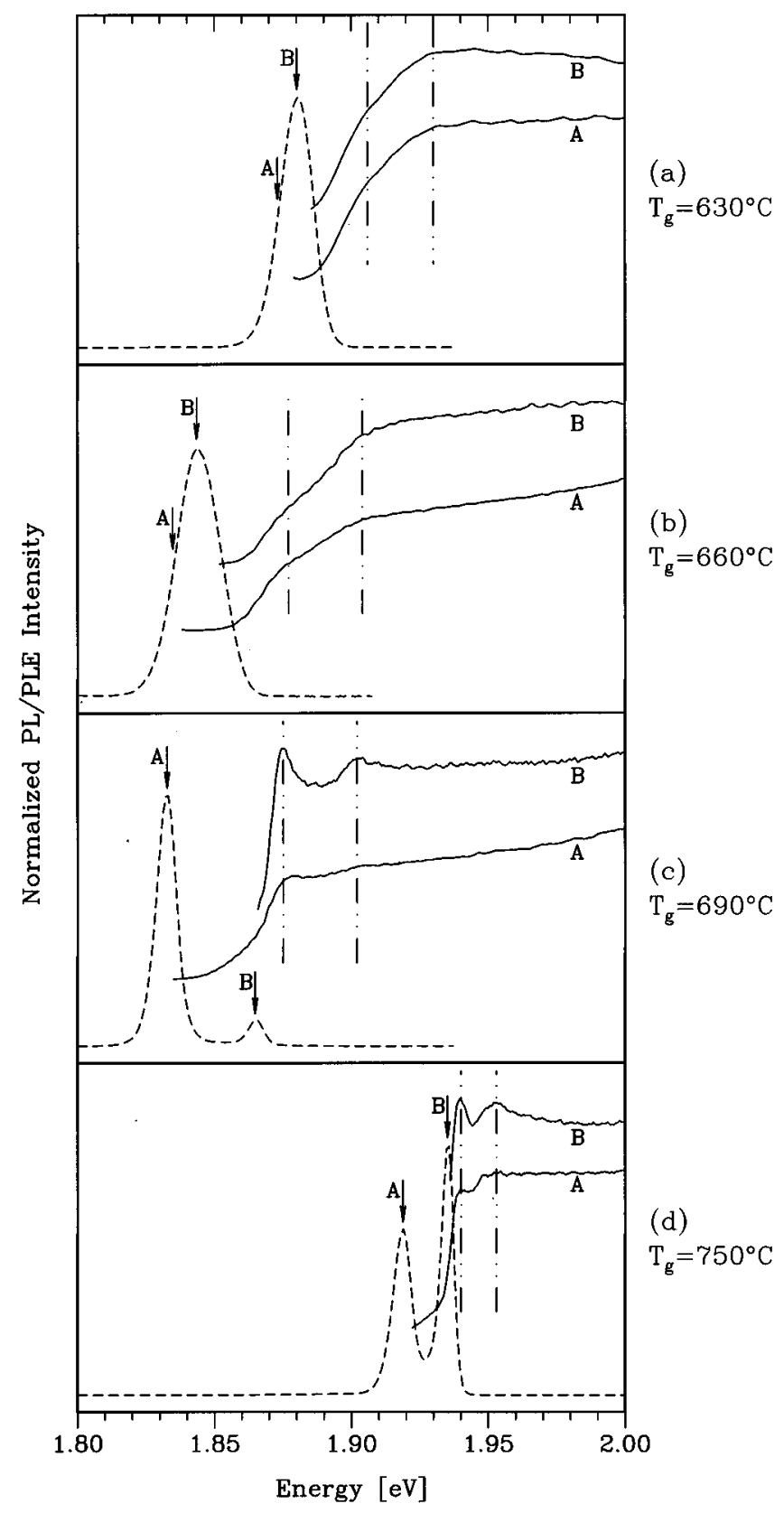

FIG. 4. PL (dashed) and PLE (solid), taken at $T=2 \mathrm{~K}$. Arrows indicate the detection energies of the corresponding PLE spectra. The order-induced valence-band splitting can be seen in the PLE spectra either by two clearly resolved excitonic peaks [(c) and (d)], or as weak shoulders in the slopes [(a) and (b)], as indicated by dashed-dotted lines. The PLE spectra are shifted on the $y$ axis.

strate (bottom). We have etched part of the epilayer to different depths on four different pieces of this sample and repeated the PL measurements on the leftover material. Etching was done by a low-damage electron-cyclotronresonance-reactive ion dry-etching process (ECR-RIE) using an $\mathrm{Ar} / \mathrm{CCl}_{2} \mathrm{~F}_{2}$ gas mixture, as described in detail in Ref. 28. The etching rate was $34 \mathrm{~nm} / \mathrm{min}$.

Figure 5 shows the result. The amplitude of the LE peak is normalized to unity in each of the spectra, and each spectrum is plotted on the same $y$ axis. Thus, the relative quantum efficiency of the direct (excitonic) recombination can be 


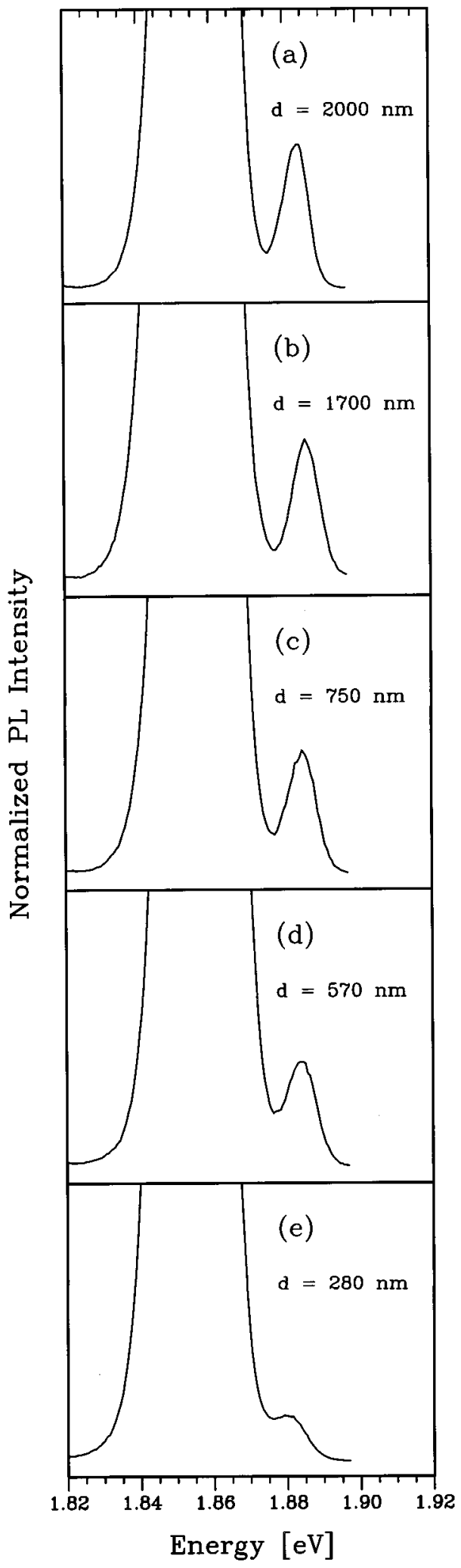

FIG. 5. PL spectra of the sample grown at $690{ }^{\circ} \mathrm{C}$, after etching a part of the epilayer. The PL of the as-grown sample is shown in (a). The remaining thickness $d$ of the layers after etching is given in each figure.

directly seen in the intensity of the HE peak. The peak ratio, as determined from spectra (a)-(e) of Fig. 5 by integrating the areas under the peaks, is plotted against the remaining layer thickness $d$ in Fig. 6. We attribute the decrease of the

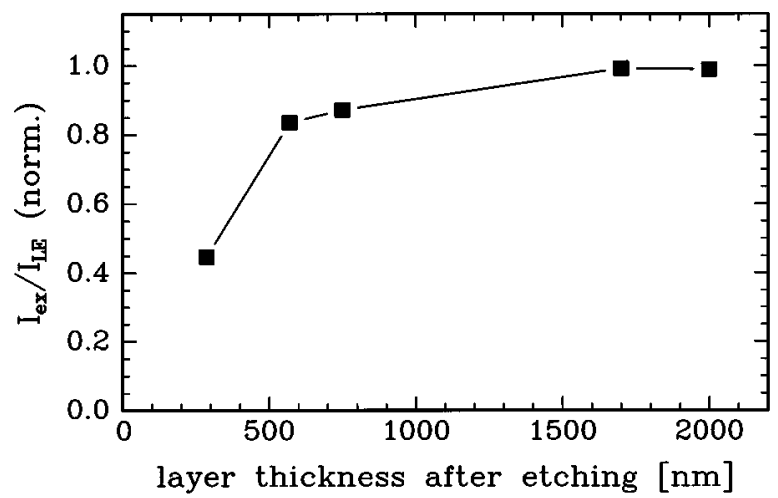

FIG. 6. Relative quantum efficiency of the direct band-band recombination at low temperature as a function of the remaining layer thickness $d$.

relative quantum efficiency of the direct recombination with decreasing layer thickness to a decrease of the average domain size in the epilayer under investigation, in analogy with the above-described results from different samples with different domain sizes.

\section{DISCUSSION}

We conclude from these experiments that the indirect transition in ordered GaInP is correlated with the domain size as determined by TEM DF images. In this section we discuss different possible explanations for the indirect transitions based on the experimental evidence gathered so far.

(1) Some experiments on ordered GaInP have been interpreted by assuming a variation in order parameter through the sample volume ${ }^{29,30}$ together with a type-II band offset between regions of different degrees of order. ${ }^{16,17,31-34}$ This situation leads to a localization of photoexcited electrons in domains of higher-order parameter, whereas holes tend to relax into regions of lower-order parameter. Indirect recombination processes between these spatially separated regions give rise to luminescence with photon energies below the fundamental band gap. This scenario is consistent with all the reported features of the low-energy emission, such as long lifetimes, absence of absorption, and shift to higher energy with increasing carrier density (due to a saturation of the transitions). ${ }^{20}$

Our experiments on the domain size also support this interpretation. If we assume that the domains that can be seen in the DF images differ slightly in their degree of order, then we expect indirect transitions to occur at domain boundaries. As the density of domain boundaries in the volume increases with decreasing domain size, we expect the indirect transition to become more important. This is actually observed. For very small domains (exciton diffusion radius or smaller), we do not even have a well-defined band gap any more, which explains the absence of the HE peak and the smeared-out PLE spectrum of our samples grown at 660 and $630^{\circ} \mathrm{C}$; however, we cannot directly prove that the LE peak comes from domain boundaries using the experiments described above. This is currently being investigated. It is also possible that there are order parameter variations on a length scale different from the one seen in the domain images. 
Then, however, this length scale is different in the four samples investigated here, in similarity with the domain sizes.

(2) In two recent theoretical articles, Mäder and Zunger report on the effects of clustering on the optical properties of III-V alloys. ${ }^{35,36}$ Besides a band-gap reduction, the authors predict (in the case of $\mathrm{GaInP}_{2}$ ) a type-II-like band alignment between $\mathrm{Ga}$ and In-rich clusters. According to these calculations, the electron wave functions should be localized on Ga-rich sites, whereas holes are localized on In-rich regions. These clusters are therefore able to act as isoelectronic traps for both electrons and holes. These traps are likely to occur with varying "binding energy." They provide another possible explanation for trapping and separation of electrons and holes and subsequent spatially indirect transition.

If clustering is the explanation of the moving LE emission, we have to conclude from our experiments that

(a) clusters are located at domain boundaries, or

(b) their volume density in different samples is correlated with the domain size.

This would have to be explained by the thermodynamics of the growth process which control both the size of ordered domains and the occurrence of compositional fluctuations, i.e., clusters.

(3) A third possibility, however, is that the LE luminescence comes directly from the domain boundaries. A close look to DF images (or high-resolution TEM images as shown in Refs. 25, 37, and 38) shows that the dark regions in TEM dark-field images are far from being just two-dimensional interfaces, although Baxter and co-workers ${ }^{37}$ and $\mathrm{Su}$ and co-workers ${ }^{25}$ have shown that (in general) antiphase boundaries are located between bright areas in DF-TEM images; however, dark regions in these images can be quite thick and fill a volume as large as that of the domains (bright regions). Up to now it is unclear whether the dark regions represent disordered material, or material of a different composition (strained), or even amorphous material. It would be consistent with the experimental results of this work, however, to assume that the material "between" ordered domains gives rise to the below-band-gap transition.

\section{SUMMARY}

We have grown a series of ordered GaInP samples on GaAs substrate misoriented $6^{\circ}$ off toward $[111]_{B}$ at different growth temperatures below and above the temperature that yields the maximum degree of order. It has been shown by DF-TEM images that the size of the ordered domains varies by as much as a factor of 50 with the growth temperature, which is the only growth parameter varied.

A low-temperature PL and PLE study on these samples reveals a close correlation between the optical properties and the domain size. The moving PL peak with photon energies below the optical band gap is found in the PL spectrum of each of the samples. A second PL peak at higher energy is attributed to the direct band-band recombination. The intensity of this peak strongly depends on the domain size. This has been shown
(1) by comparing PL spectra of different samples with a different average domain size, and

(2) by comparing PL spectra taken from one sample after removing a part of the epilayer from the top.

This also results in a different domain size in the investigated volume, because the domains become smaller when going closer to the substrate.

Samples with smaller ordered domains are shown to have a weaker, even completely vanishing, direct recombination peak in PL and a smeared-out band edge as measured by PLE, with no excitonic resonances.

These results have been discussed with regard to possible explanations of the origin of the moving emission. Further experiments, however, are needed to make definite conclusions.

\section{ACKNOWLEDGMENTS}

We would like to thank Yong Zhang and F. Driessen for helpful discussions, E. Kohler for assistance in MOVPE growth, and C. Eyermann and B. Höhing for dry etching. Part of this work was supported by the Landesprogramm "Höchstfrequenzgrenzen in der Optoelektronik," and by ESPRIT Project No. 6135 (HIRED).

${ }^{1}$ Y. Ueno, H. Fuji, K. Kobayashi, K. Endo, A. Gomyo, K. Hara, S. Kawata, T. Yuasa, and T. Suzuki, Jpn. J. Appl. Phys. 29, L1666 (1990).

${ }^{2}$ K. Itaya, M. Ishikawa, G. Hatakoshi, and Y. Uematsu, IEEE J. Quantum Electron. QE-27, 1496 (1991).

${ }^{3}$ G. G. Forstmann, F. Barth, H. Schweizer, M. Moser, C. Geng, F. Scholz, and E. P. O'Reilly, Semicond. Sci. Technol. 9, 1268 (1994).

${ }^{4}$ D. J. Friedman, S. R. Kurtz, K. A. Bertness, A. E. Kibbler, C. Kramer, J. M. Olson, D. L. King, B. R. Hansen, and J. K. Snyder, Prog. Photovolt. Res. Appl. 3, 47 (1995).

${ }^{5}$ A. Zunger and S. Mahajan, in Handbook on Semiconductors, edited by T. S. Moss and S. Mahajan (Elsevier, Amsterdam, 1994), Vol. 3, p. 1399.

${ }^{6}$ S.-H. Wei and A. Zunger, Phys. Rev. B 39, 3279 (1989).

${ }^{7}$ S.-H. Wei and A. Zunger, Appl. Phys. Lett. 56, 662 (1990).

${ }^{8}$ S.-H. Wei and A. Zunger, Phys. Rev. B 49, 14337 (1994).

${ }^{9}$ A. Mascarenhas, S. Kurtz, A. Kibbler, and J. M. Olson, Phys. Rev. Lett. 63, 2108 (1989).

${ }^{10}$ D. J. Mowbray, R. A. Hogg, M. S. Skolnick, M. C. DeLong, S. R. Kurtz, and J. M. Olson, Phys. Rev. B 46, 7232 (1992).

${ }^{11}$ T. Kanata, M. Nishimoto, H. Nakayama, and T. Nishino, Phys. Rev. B 45, 6637 (1992).

${ }^{12}$ G. S. Horner, A. Mascarenhas, R. G. Alonso, D. J. Friedman, K. Sinha, K. A. Bertness, J. G. Zhu, and J. M. Olson, Phys. Rev. B 48, 4944 (1993).

${ }^{13}$ R. G. Alonso, A. Mascarenhas, S. Froyen, G. S. Horner, K. Bertness, and J. M. Olson, Solid State Commun. 85, 1021 (1993).

${ }^{14}$ P. Ernst, C. Geng, F. Scholz, H. Schweizer, Y. Zhang, and A. Mascarenhas, Appl. Phys. Lett. 67, 2347 (1995).

${ }^{15}$ M. Kondow and S. Minagawa, Appl. Phys. Lett. 54, 1760 (1989).

${ }^{16}$ J. E. Fouquet, V. M. Robbins, J. Rosner, and O. Blum, Appl. Phys. Lett. 57, 1566 (1990).

${ }^{17}$ M. C. DeLong, W. D. Ohlsen, I. Viohl, P. C. Taylor, and J. M. Olson, J. Appl. Phys. 70, 2780 (1991).

${ }^{18}$ M. C. DeLong, D. J. Mowbray, R. A. Hogg, M. S. Skolnick, M. Hopkinson, J. P. R. David, P. C. Taylor, S. R. Kurtz, and J. M. Olson, J. Appl. Phys. 73, 5163 (1993).

${ }^{19}$ F. A. J. M. Driessen, G. J. Bauhuis, S. M. Olsthoorn, and L. J. Giling, Phys. Rev. B 48, 7889 (1993).

${ }^{20}$ P. Ernst, C. Geng, F. Scholz, and H. Schweizer, Phys. Status Solidi B (to be published).

${ }^{21}$ J. Chevallier and A. Laugier, Phys. Status Solidi A 8, 437 (1971).

${ }^{22}$ E. Zacks and A. Halperin, Phys. Rev. B 6, 3072 (1972).

${ }^{23}$ M. Kondow, H. Kakibayashi, and S. Minegawa, J. Cryst. Growth 88, 291 (1988). 
${ }^{24}$ P. Bellon, J. P. Chevalier, E. Augarde, J. P. André, and G. P. Martin, J. Appl. Phys. 66, 2388 (1989).

${ }^{25}$ L. C. Su, I. H. Ho, and G. B. Stringfellow, J. Appl. Phys. 75, 5135 (1994).

${ }^{26}$ L. C. Su, I. H. Ho, and G. B. Stringfellow, J. Appl. Phys. 76, 3520 (1994).

${ }^{27}$ D. M. Follstaedt, R. P. Schneider, Jr., and E. D. Jones, J. Appl. Phys. 77, 3077 (1995)

${ }^{28}$ J. Hommel, F. Schneider, M. Moser, C. Geng, F. Scholz, and H. Schweizer, Microelectron. Eng. 23, 349 (1994)

${ }^{29}$ G. S. Horner, A. Mascarenhas, R. G. Alonso, S. Froyen, K. A. Bertness, and J. M. Olson, Phys. Rev. B 49, 1727 (1994).

${ }^{30}$ K. Sinha, A. Mascarenhas, G. S. Horner, K. A. Bertness, S. R. Kurtz, and J. M. Olson, Phys. Rev. B 50, 7509 (1994).

${ }^{31}$ R. P. Schneider, Jr., E. D. Jones, and D. M. Follstaedt, Appl. Phys. Lett. 65, 587 (1994).
${ }^{32}$ E. D. Jones, D. M. Follstaedt, H. Lee, J. S. Nelson, and R. P. Schneider, Jr., in 22nd International Conference on the Physics of Semiconductors, edited by D. J. Lockwood (World Scientific, Singapore, 1995).

${ }^{33}$ J. E. Fouquet, M. S. Minsky, and S. J. Rosner, Appl. Phys. Lett. 63, 3212 (1993).

${ }^{34}$ C. Geng, P. Ernst, D. Haase, G. Hahn, F. Phillipp, A. Dörnen, H. Schweizer, and F. Scholz, in 6th European Workshop on MOVPE, Gent, Belgium, 1995.

${ }^{35}$ K. A. Mäder and A. Zunger, Appl. Phys. Lett. 64, 2882 (1994).

${ }^{36}$ K. A. Mäder and A. Zunger, Phys. Rev. B 51, 10462 (1995).

${ }^{37}$ C. S. Baxter, W. M. Stobbs, and J. H. Wilkie, J. Cryst. Growth 112, 373 (1991).

${ }^{38}$ F. A. J. M. Driessen, G. J. Bauhuis, P. R. Hageman, A. van Geelen, and L. J. Giling, Phys. Rev. B 50, 17105 (1994). 